\title{
Prognostic value of low-dose dobutamine stress echocardiography in patients with aortic stenosis and impaired left ventricular function
}

\author{
Edyta Płońska-Gościniak ${ }^{1}$, Piotr Lipiec², Patrizio Lancellotti ${ }^{3}$, Andrzej Szyszka ${ }^{4}$ Zbigniew Gąsior ${ }^{5}$, \\ Ilona Kowalik ${ }^{6}$, Andrzej Gackowski , Piotr Gościniak ${ }^{8}$ Karina Wierzbowska-Drabik², \\ Jarosław D. Kasprzak²
}

\author{
1Department of Cardiology, Pomeranian Medical University, Szczecin, Poland \\ 2Department of Cardiology, Medical University of Lodz, Poland \\ ${ }^{3}$ Department of Cardiology, Heart Valve Clinic, University of Liège, Hospital Sart \\ Tilman, Liège, Belgium \\ ${ }^{4}$ Department of Cardiology, Medical University of Poznan, Poland \\ ${ }^{5}$ Department of Cardiology, Medical University of Silesia, Katowice, Poland \\ 6 Institute of Cardiology, Warsaw, Poland \\ 7Department of Coronary Disease, Jagiellonian University Medical College, Krakow, \\ Poland \\ 8Department of Cardiology, Voivodeship Hospital, Szczecin, Poland
}

Submitted: 28 May 2012

Accepted: 11 January 2013

Arch Med Sci 2013; 9, 3: 434-439

DOI: 10.5114 /aoms.2013.35422

Copyright (c) 2013 Termedia \& Banach

\section{Abstract}

Introduction: The aim of this multicenter, prospective study was to evaluate the long-term prognostic value of low-dose dobutamine stress echocardiography (LDDSE) in patients with aortic stenosis (AS) and depressed left ventricular (LV) function.

Material and methods: The study group comprised 39 patients (34 male, mean age $59 \pm 13$ years) with AS (peak gradient $>25 \mathrm{~mm} \mathrm{Hg}$ ), LV ejection fraction (LVEF) $\leq 45 \%$ and low transaortic gradient (peak gradient $\leq 45 \mathrm{~mm} \mathrm{Hg}$, mean gradient $\leq 35 \mathrm{~mm} \mathrm{Hg}$ ). The qualification for subsequent therapeutic procedures was based on generally accepted indications. All patients underwent LDDSE and coronary angiography. Twelve months after LDDSE patients underwent control resting echocardiography and clinical evaluation.

Results: Twenty-seven (69.2\%) patients had preserved contractile reserve. In this subgroup, true-severe AS was diagnosed in 12 patients, whereas pseudosevere AS was found in 15 patients. Nine patients with true-severe AS, 2 patients with pseudo-severe AS and 7 patients without contractile reserve were referred for surgical treatment. The independent risk factors of death during follow-up were: aortic valve area (AVA) at peak stress $<0.8 \mathrm{~cm}^{2}(\mathrm{OR} 1.4 ; p=0.003)$ and LVEF at rest $<35 \%$ (OR 6.8; $p=0.05)$. The independent risk factors of composite end-point (death or myocardial infarctions or pulmonary edema) were: AVA at stress $<0.8 \mathrm{~cm}^{2}$ (OR 4.0; $\left.p=0.03\right)$, absence of AVA increase during LDDSE (OR 5.7; $p=0.005)$, absence of contractile reserve (OR $4.5 ; p=0.01)$ and presence of significant CAD (OR 6.9; $p=0.02$ ).

Conclusions: In patients with AS and depressed LVEF, LDDSE is a useful tool for long-term risk stratification.

Key words: stress echocardiography, aortic stenosis, left ventricular dysfunction, coronary artery disease, prognosis, aortic valve replacement.

\author{
Corresponding author: \\ Prof. Edyta Płońska-Gościniak \\ $\mathrm{MD}, \mathrm{PhD}$ \\ Department of Cardiology \\ Pomeranian Medical School \\ 72 Powstańców \\ Wielkopolskich St \\ 70-111 Szczecin, Poland \\ Phone: +48 914661252 \\ Fax: +48914661379 \\ E-mail: edytaplonska@life.pl
}




\section{Introduction}

Aortic stenosis (AS) with coexisting left ventricular (LV) dysfunction presents both diagnostic and therapeutic challenges. In this scenario, lowdose dobutamine stress echocardiography (LDDSE) enables the detection of contractile reserve [1] Based on LDDSE results patients with preserved LV contractile reserve can be further classified into at least two important subgroups: those with truesevere AS, who will benefit from surgical treatment, and those with pseudo-severe AS, who can remain on medical treatment $[2,3]$. Other treatment options, such as transcatheter aortic valve implantation or cardiac resynchronization therapy, have also been recently described in these patients [4-6] The choice of the best approach in patients without contractile reserve remains a matter of debate [7].

Stress echocardiography has been demonstrated to have prognostic value in patients with AS and impaired LV function [8, 9]. However, the available data regarding utility of LDDSE as a tool for longterm risk stratification are still limited. The aims of this multicenter and prospective study were: to evaluate the long-term prognostic value of LDDSE in patients with AS and depressed LV function with regard to clinical and echocardiographic outcome variables, and to compare the effects of treatment strategy on the LV remodeling process.

\section{Material and methods}

\section{Patient population}

We prospectively studied 39 patients with AS (peak gradient $>25 \mathrm{~mm} \mathrm{Hg}$ ), depressed LV systolic function (LV ejection fraction, LVEF $\leq 45 \%$ ) and low transaortic pressure gradient (peak gradient $\leq 45 \mathrm{~mm} \mathrm{Hg}$ and at the same time mean gradient $\leq 35 \mathrm{~mm} \mathrm{Hg}$ ). Exclusion criteria included: chronic atrial fibrillation, other significant valve disease, moderate or severe aortic regurgitation, contraindications to LDDSE, clinical and hemodynamic instability, implanted pacemaker and poor quality of echocardiographic images at rest precluding reliable assessment of LV contractility, valve morphology and function.

\section{Study protocol}

All patients underwent LDDSE. Patients were instructed to abstain from $\beta$-blockers and calcium-antagonists for at least $24 \mathrm{~h}$ before the test. All other medications were continued as prescribed. The qualification for subsequent coronary angiography and further therapeutic procedures was based on generally accepted indications and was a decision of attending physicians, not influenced by the study protocol. Twelve months after
LDDSE patients underwent control resting echocardiography and clinical evaluation. The clinical end-points included: death, myocardial infarction and significant worsening of heart failure symptoms (pulmonary edema). The study protocol was approved by the Ethics Committee of our institution and written consent was obtained from all participants.

\section{Low-dose dobutamine stress echocardiography}

The standard LDDSE protocol was used for stress echocardiography. The dobutamine dose sequence was $5 \mu \mathrm{g} / \mathrm{kg} / \mathrm{min}$ and $10 \mu \mathrm{g} / \mathrm{kg} / \mathrm{min}$, each maintained for 3 min with an infusion pump.

Echocardiographic images were acquired at each stage of LDDSE (Sonos 2500 and Sonos 5500). At each stage transaortic peak and mean gradients were measured and aortic valve area (AVA) was determined using the continuity equation. Left ventricular wall motion was assessed visually and scored in each of the 16 segments as normal (1), hypokinetic (2), akinetic (3) or dyskinetic (4). Poorly visible segments were excluded from the analysis. Moreover, at each stage LVEF was measured using biplane Simpson's method.

Based on the difference between peak and baseline EF the patients were classified as having preserved contractile reserve ( $\geq 20 \%$ increase in LVEF) or having no contractile reserve $(<20 \%$ increase in LVEF). In the subgroup with preserved contractile reserve the patients with AVA increase during LDDSE $\leq 0.3 \mathrm{~cm}^{2}$ and AVA at peak dose of dobutamine $\leq 1 \mathrm{~cm}^{2}$ were classified as having true-severe AS, whereas the patients with $>0.3 \mathrm{~cm}^{2}$ increase in AVA during LDDSE or AVA at peak dose of dobutamine $>1 \mathrm{~cm}^{2}$ were classified as having pseudosevere AS.

\section{Statistical analysis}

Continuous and categorical variables are expressed as mean \pm SD and as percentages (\%), respectively. Group comparisons were obtained for categorical variables with $\chi^{2}$ test and for continuous variables with one-way analysis of variance. Assessment of time-to-event data was conducted by Kaplan-Meier method and survival of different groups was compared using the log-rank test. Additionally, the odds ratio (OR) was calculated to assess the risk of particular events. Effect of covariates on outcomes was determined using Cox proportional hazard regression in a stepwise manner. Clinically relevant variables with a $p$ value $<0.1$ on univariable analysis were incorporated into the multivariable models. Value of $p$ of less than 0.05 was considered to indicate statistical significance. 


\section{Results}

\section{Characteristics of the patients}

Patients' characteristics are summarized in Table I. The LDDSE was well tolerated - there were no major adverse events. The baseline echocardiographic data and results of LDDSE are presented in Table II. There were 27 (69.2\%) patients with preserved contractile reserve and 12 (30.8\%) patients without contractile reserve. In the subgroup with preserved contractile reserve true-severe AS was diagnosed in 12 patients, whereas pseudo-severe AS was found in 15 patients.

All patients underwent coronary angiography. Significant coronary artery disease (CAD) defined as $\geq 50 \%$ stenosis was diagnosed in 21 (53.8\%) patients, including 7 (18\%) patients with single-vessel disease, 7 (18\%) patients with two-vessel disease and 7 (18\%) patients with three-vessel disease.

\section{Treatment strategy}

All (12) patients with true severe AS were referred for surgical treatment. However, two of them did not consent to surgery and one died before surgery.

Table I. Study population characteristics $(n=39)$

\begin{tabular}{|c|c|}
\hline Parameter & Results \\
\hline Age [years] & $59 \pm 13$ \\
\hline Male & $34(87.2 \%)$ \\
\hline Weight [kg] & $77 \pm 11$ \\
\hline Height $[\mathrm{cm}]$ & $172 \pm 7$ \\
\hline Body mass index $\left[\mathrm{kg} / \mathrm{m}^{2}\right]$ & $26 \pm 3$ \\
\hline Body surface area [m²] & $1.90 \pm 0.15$ \\
\hline Dyslipidemia & $18(46.2 \%)$ \\
\hline Diabetes & $4(10.3 \%)$ \\
\hline Hypertension & $16(41.0 \%)$ \\
\hline Smoking history & $13(33.3 \%)$ \\
\hline Previous myocardial infarction & $4(10.3 \%)$ \\
\hline Dyspnea at rest & $10(25.6 \%)$ \\
\hline Peripheral edema & $4(10.3 \%)$ \\
\hline Fatigue & $25(64.1 \%)$ \\
\hline History of angina & $14(35.9 \%)$ \\
\hline Ventricular arrhythmia & $4(10.3 \%)$ \\
\hline Dyspnea at exertion & $25(64.1 \%)$ \\
\hline Atypical chest pain & $8(20.5 \%)$ \\
\hline Syncope & $7(17.9 \%)$ \\
\hline Vertigo & $1(2.6 \%)$ \\
\hline NYHA I & $5(12.8 \%)$ \\
\hline NYHA II & $18(46.2 \%)$ \\
\hline NYHA III & $16(41.0 \%)$ \\
\hline
\end{tabular}

Data are presented as the mean value \pm SD or number (\%) of patients
Therefore, 9 patients underwent aortic valve replacement (AVR); two of them had concomitant coronary artery bypass grafting (CABG).

Thirteen patients with pseudo-severe AS were treated pharmacologically. One of them underwent percutaneous coronary intervention during the follow-up period. The remaining 2 patients were referred for surgical treatment due to the presence of one criterion of true severe AS (either $\leq 0.3 \mathrm{~cm}^{2}$ increase in AVA during LDDSE or AVA at peak dose of dobutamine $\leq 1 \mathrm{~cm}^{2}$ ).

Within the subgroup without contractile reserve, 7 patients underwent AVR (5 of them had concomitant $(A B G)$ and 5 patients were treated pharmacologically.

\section{Clinical follow-up}

During the follow-up period (mean duration 353 \pm 38 days), there were 4 deaths, 3 myocardial infarctions ( 1 fatal) and 3 cases of pulmonary edema. One death occurred in a patient with true severe AS awaiting surgery; there were 2 peri-operative deaths (one in a patient with true-severe AS and one in a patient without contractile reserve) and one death in a patient without contractile reserve who underwent AVR.

The independent risk factors of death were: AVA at peak stress < $0.8 \mathrm{~cm}^{2}$ (OR 1.4; 95\% Cl 1.0-2.08; $p=0.003)$ and LVEF at rest $<35 \%($ OR $6.8 ; 95 \% \mathrm{Cl}$ 1.0-58.4; $p=0.05$ ).

The independent risk factors of composite endpoint (death or myocardial infarctions or pulmonary edema) were: AVA at peak stress $<0.8 \mathrm{~cm}^{2}$ (OR 4.0; 95\% Cl 1.2-13.5; $p=0.03)$, absence of AVA increase during LDDSE (OR 5.7; 95\% Cl 2.0-16.0; $p=0.005)$, absence of contractile reserve (OR $4.5 ; 95 \% \mathrm{Cl} 1.3$ $15.0 ; p=0.01)$ and presence of significant CAD (OR 6.9; 95\% Cl 1.0-50.0; $p=0.02$ ) (Figure 1).

Table II. Baseline echocardiographic data and results of LDDSE

\begin{tabular}{|lccc|}
\hline Parameter & Rest & Peak stress & Value of $p$ \\
\hline Heart rate [bpm] & $76 \pm 12$ & $82 \pm 12$ & 0.001 \\
\hline $\begin{array}{l}\text { Systolic blood } \\
\text { pressure [mm Hg] }\end{array}$ & $136 \pm 24$ & $137 \pm 19$ & 0.004 \\
\hline $\begin{array}{l}\text { Diastolic blood } \\
\text { pressure [mm Hg] }\end{array}$ & $84 \pm 11$ & $84 \pm 11$ & NS \\
\hline LV EF [\%] & $39 \pm 8$ & $45.3 \pm 10$ & 0.001 \\
\hline AVA [cm²] & $0.8 \pm 0.2$ & $0.99 \pm 0.29$ & $\mathrm{NS}$ \\
\hline $\begin{array}{l}\text { Mean aortic } \\
\text { gradient [mm Hg] }\end{array}$ & $24.0 \pm 5.5$ & $31.8 \pm 8.5$ & 0.001 \\
\hline $\begin{array}{l}\text { Peak aortic } \\
\text { gradient [mm Hg] }\end{array}$ & $37.5 \pm 6.4$ & $52 \pm 14.2$ & 0.001 \\
\hline Aortic Vmax [m/s] & $3.11 \pm 0.4$ & $3.57 \pm 0.49$ & 0.01 \\
\hline
\end{tabular}

Data are presented as the mean value $\pm S D$. AVA-aortic valve area, $E F$ - ejection fraction, $L V$ - left ventricle 
All survivors with true-severe AS who underwent surgery $(n=8)$ improved in their functional status by one grade according to NYHA classification. Within the group of survivors without contractile reserve who underwent surgery $(n=5) 2$ patients remained in the same NYHA class, 1 patient's status improved by one class, and 2 patients' status worsened by one class.

\section{Echocardiographic follow-up}

Changes in echocardiographic parameters of the LV and aortic valve observed after 12 months in the study group are presented in Table III. The changes in LVEDD, LVEF and wall motion score index (WMSI) differed significantly between the patients treated surgically and pharmacologically ( $p=0.005, p=0.003$, and $p=0.02$, respectively).

In patients treated pharmacologically the increase in LVEDD after 12 months correlated with baseline maximum velocity at rest $(r=0.73, p<0.01)$ and with WMSI at peak stress $(r=0.52, p<0.05)$, whereas the increase in peak and mean gradients after 12 months correlated with maximum velocity at peak stress and peak and mean gradients at peak stress $(r=0.48-0.77 ; p=0.001-0.05)$. When baseline clinical characteristics were taken into consideration, we found that the increase in LVEDD was significantly more pronounced in patients aged $\geq 65$ years $(p<0.05)$.

In patients treated surgically the change in LVEDD after 12 months correlated with baseline maximum velocity at rest $(r=0.74, p<0.01)$, peak gradient at rest $(r=0.7, p<0.05)$ and WMSI at rest $(r=0.66, p<0.05)$. When baseline clinical characteristics were taken into consideration, we found that the improvement in LVEF was significantly less pronounced in patients with CAD and patients with hypertension $(p<0.05)$.

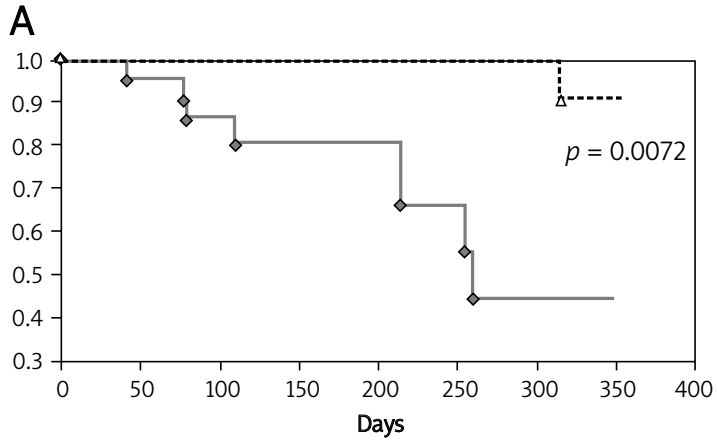

- CAD+ --.-..CAD-

\section{B}

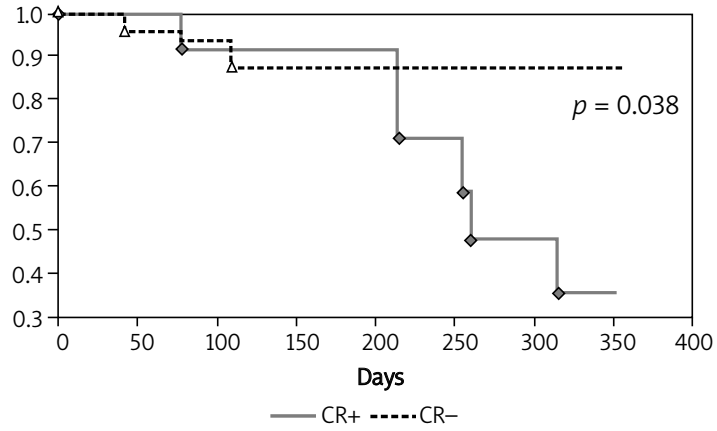

C

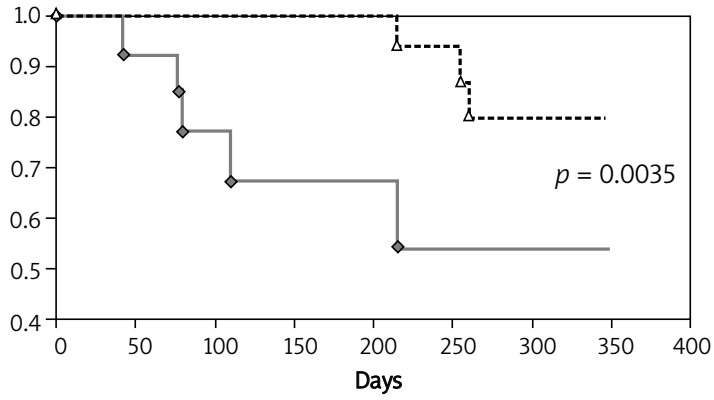

- AVA at stress $<0.8 \mathrm{~cm}^{2} \ldots$....AVA at stress $\geq 0.8 \mathrm{~cm}^{2}$

Figure 1. Kaplan-Meier curves for survival free from composite end-point (death or myocardial infarctions or pulmonary edema) in regard to presence of significant CAD $(A)$, contractile reserve $(C R)$ observed at LDDSE (B) and AVA at peak stress (C)

Table III. Changes in echocardiographic parameters of the left ventricle and aortic valve observed after 12 months in patients treated surgically and pharmacologically, with and without contractile reserve on LDDSE

\begin{tabular}{|c|c|c|c|c|c|c|c|}
\hline \multirow{2}{*}{$\begin{array}{l}\text { Changes } \\
\text { after } \\
1 \text { year }\end{array}$} & \multirow{2}{*}{$\begin{array}{c}\text { All } \\
\text { patients }\end{array}$} & \multicolumn{3}{|c|}{ Pharmacological treatment } & \multicolumn{3}{|c|}{ Surgical treatment } \\
\hline & & $\begin{array}{c}\text { All } \\
(n=20)\end{array}$ & $\begin{array}{l}\text { No contractile } \\
\text { reserve } \\
(n=5)\end{array}$ & $\begin{array}{l}\text { Contractile } \\
\text { reserve } \\
(n=15)\end{array}$ & $\begin{array}{c}\text { All } \\
(n=15)\end{array}$ & $\begin{array}{l}\text { No contractile } \\
\text { reserve } \\
(n=5)\end{array}$ & $\begin{array}{c}\text { Contractile } \\
\text { reserve } \\
(n=10)\end{array}$ \\
\hline$\Delta$ LVEF & $1.1 \pm 4.5$ & $-0.9 \pm 3.4^{\star}$ & $-2.6 \pm 2.0$ & $-0.3 \pm 0.7$ & $3.40 \pm 4.70^{*}$ & $1.6 \pm 5.1$ & $4.9 \pm 4.1$ \\
\hline$\Delta$ LVEDD & $-0.0 \pm 4.6$ & $1.96 \pm 3.64^{\dagger}$ & $3.70 \pm 4.1$ & $1.34 \pm 0.9$ & $-2.26 \pm 4.72^{\dagger}$ & $-2.4 \pm 3.8$ & $-2.2 \pm 5.5$ \\
\hline$\triangle \mathrm{AVA}$ & $0.30 \pm 0.59$ & $-0.04 \pm 0.06$ & $5-0.04 \pm 0.06$ & $-0.04 \pm 0.06$ & $0.91 \pm 0.64$ & $0.90 \pm 0.94$ & $0.93 \pm 0.32$ \\
\hline$\Delta$ WMSI & $0.05 \pm 0.20$ & $0.12 \pm 0.19^{\ddagger}$ & $0.17 \pm 0.11$ & $0.10 \pm 0.20$ & $-0.04 \pm 0.18^{\ddagger}$ & $0.08 \pm 0.08^{a}$ & $-0.11 \pm 0.19^{a}$ \\
\hline$\Delta$ Mean aortic gradient & t $0.3 \pm 8.3$ & $4.4 \pm 6.8$ & $3.5 \pm 2.5$ & $4.7 \pm 2.0$ & $-6.6 \pm 6.0$ & $-4.9 \pm 6.8$ & $-8.1 \pm 5.4$ \\
\hline$\Delta$ Max aortic gradient & $-1.0 \pm 12.8$ & $6.4 \pm 11.6$ & $3.8 \pm 5.2$ & $7.3 \pm 3.2$ & $-8.3 \pm 9.0$ & $-5.5 \pm 8.5$ & $-10.6 \pm 9.6$ \\
\hline$\Delta V \max$ & $-0.06 \pm 0.52$ & $0.20 \pm 0.37$ & $0.18 \pm 0.21$ & $0.20 \pm 0.10$ & $-0.47 \pm 0.47$ & $-0.33 \pm 0.39$ & $-0.59 \pm 0.54$ \\
\hline
\end{tabular}




\section{Discussion}

Our study shows that in patients with AS and depressed LVEF, LDDSE is a useful tool for long-term risk stratification. Low LVEF at rest and low AVA at peak stress were identified as independent risk factors of death, whereas small AVA at peak stress, lack of increase in AVA during test, absence of contractile reserve and presence of CAD were independent risk factors of composite end-point (death or myocardial infarctions or pulmonary edema) in long-term follow-up. Moreover, regardless of the nature of AS, patients treated medically present an ongoing LV remodeling process, whereas surgery is accompanied by a reverse LV remodeling process in survivors.

In our study the best results were obtained in surgically treated patients with true-severe AS and preserved LV contractile reserve - the perioperative mortality in this group was $11 \%$ with no further deaths in the follow-up period. The patients who had true-severe AS and were not operated on were more likely to die (33\%). However, in patients without contractile reserve the perioperative mortality was high (29\%) and similar to the value reported by Tribouilloy et al. [10] In echocardiographic followup the best results were obtained in patients with preserved contractile reserve who were treated surgically.

The first multicenter study to demonstrate a lack of contractile reserve on LDDSE as a risk factor of operative and long-term mortality in patients with low-flow low-gradient AS was published by Monin et al. [8]. This finding was confirmed by other reports, including the multicenter TOPAS study, which also indicated impaired functional capacity as measured by the Duke Activity Status Index or 6-minute walk test distance and more severe valve stenosis as measured by projected AVA at a normal transvalvular flow rate as other significant risk factors of poor outcome [9]. The assessment of contractile reserve by dobutamine challenge has been proved clinically useful also in combination with invasive assessment of cardiac output and aortic pressure gradients [11]. Therefore, in the most recent guidelines on stenosis assessment it was underlined that absence of contractile reserve (failure to increase stroke volume or EF by $20 \%$ of baseline value) is a predictor of poor outcome [3]. At the same time, the authors of the guidelines felt that the prognostic significance of all other parameters obtained by means of LDDSE needs to be further examined before they can be included in recommendations [3]. Our study addressed these concerns by providing further data on the matter.

We observed a significant increase in LVEF in patients treated surgically and it was related to the presence of significant CAD. It is concurrent with results of previous studies $[12,13]$. However, the relationship between hypertension and change in LVEF observed in our study has not been demonstrated before. In our group, presence of CAD was also an independent risk factor of composite endpoint (death or myocardial infarctions or pulmonary edema), but it was not significantly related to mortality. Similar results (no influence on survival) were obtained by other investigators, but at the same time previous myocardial infarction and previous CABG were identified as independent predictors of mortality $[9,13,14]$.

In patients treated pharmacologically a decrease in LVEF over a 12-month follow-up period did not reach statistical significance, but at the same time we observed a significant increase in LVEDD - it was more pronounced in elderly patients, with higher transaortic maximum velocity at rest and with more wall motion abnormalities (higher WMSI) at stress. Moreover, the peak and mean gradients at stress predicted the progression of stenosis severity (the increase in peak and mean gradients).

The main limitations of our study include the relatively small size of the group and controversies that may arise around our inclusion criteria (AS with LVEF $\leq 45 \%$, peak transaortic gradient $\leq 45 \mathrm{~mm} \mathrm{Hg}$, mean transaortic gradient $\leq 35 \mathrm{~mm} \mathrm{Hg}$ ). However, the cutoff values are close to recently published guidelines, which recommend the following definition for lowflow low gradient AS: effective orifice area $<1.0 \mathrm{~cm}^{2}$, LVEF $<40 \%$ and mean pressure gradient $<30-40$ $\mathrm{mm} \mathrm{Hg}$ [3]. It must be noted that the definitions and inclusion criteria used by different investigators studying patients with AS and depressed LVEF varied greatly in the literature available at the time of designing our study $[8,11,13,15]$. Patients were followed up for a relatively short period of time (12 months) and we only considered clinical and echocardiographic end-points. Another limitation is a lack of patients undergoing other treatment techniques such as transcatheter aortic valve implantation or cardiac resynchronization therapy.

In conclusion, we demonstrated that LDDSE serves to determine contractility reserve of the LV and severity of AS and thus is useful for prognostication in patients with AS and severe systolic dysfunction of the LV. Benefits of surgery are highest in the patients with preserved contractility reserve and true-severe AS.

\section{References}

1. Sicari R, Nihoyannopoulos P, Evangelista A, et al.; European Association of Echocardiography. Stress echocardiography expert consensus statement: European Association of Echocardiography (EAE) (a registered branch of the ESC). Eur J Echocardiogr 2008; 9: 415-37.

2. Vahanian A, Baumgartner H, Bax J, et al.; Task Force on the Management of Valvular Hearth Disease of the European Society of Cardiology; ESC Committee for 
Practice Guidelines. Guidelines on the management of valvular heart disease: The Task Force on the Management of Valvular Heart Disease of the European Society of Cardiology. Eur Heart J 2007; 28: 230-68.

3. Baumgartner H, Hung J, Bermejo J, et al.; American Society of Echocardiography; European Association of Echocardiography. Echocardiographic assessment of valve stenosis: EAE/ASE recommendations for clinical practice. Eur J Echocardiogr 2009; 10: 1-25.

4. Clavel MA, Webb JG, Rodés-Cabau J, et al. Comparison between transcatheter and surgical prosthetic valve implantation in patients with severe aortic stenosis and reduced left ventricular ejection fraction. Circulation 2010; 122: 1928-36.

5. Czerwinska K, Orłowska-Baranowska E, Witkowski A, et al. Transapical aortic valve implantation - a rescue procedure for patients with aortic stenosis and "porcelain aorta". Arch Med Sci 2011; 7: 528-32.

6. Konstantino Y, Zimetbaum PJ, Hsing J, Kramer DB, Chang JD. Cardiac resynchronization therapy for low-flow, low-gradient aortic stenosis. Eur J Heart Fail 2010; 12: 889-92.

7. Bermejo J, Yotti R. Low-gradient aortic valve stenosis: value and limitations of dobutamine stress testing. Heart 2007; 93: 298-302

8. Monin JL, Quéré JP, Monchi M, et al. Low-gradient aortic stenosis: operative risk stratification and predictors for long-term outcome: a multicenter study using dobutamine stress hemodynamics. Circulation 2003; 108: 319-24.

9. Clavel MA, Fuchs C, Burwash IG, et al. Predictors of outcomes in low-flow, low-gradient aortic stenosis: results of the multicenter TOPAS Study. Circulation 2008; 118: S234-42.

10. Tribouilloy C, Lévy F, Rusinaru D, et al. Outcome after aortic valve replacement for low-flow/low-gradient aortic stenosis without contractile reserve on dobutamine stress echocardiography. J Am Coll Cardiol 2009; 53: 1865-73.

11. Nishimura RA, Grantham JA, Connolly HM, Schaff HV, Higano ST, Holmes DR Jr. Low-output, low-gradient aortic stenosis in patients with depressed left ventricular systolic function: the clinical utility of the dobutamine challenge in the catheterization laboratory. Circulation 2002; 106: 809-13.

12. Quere JP, Monin JL, Levy F, et al. Influence of preoperative left ventricular contractile reserve on postoperative ejection fraction in low-gradient aortic stenosis. Circulation 2006; 113: 1738-44.

13. Powell DE, Tunick PA, Rosenzweig BP, et al. Aortic valve replacement in patients with aortic stenosis and severe left ventricular dysfunction. Arch Intern Med 2000; 160: 1337-41.

14. Levy F, Laurent M, Monin JL, et al. Aortic valve replacement for low-flow/low-gradient aortic stenosis operative risk stratification and long-term outcome: a European multicenter study. J Am Coll Cardiol 2008; 51: 1466-72.

15. Schwammenthal E, Vered Z, Moshkowitz Y, et al. Dobutamine echocardiography in patients with aortic stenosis and left ventricular dysfunction: predicting outcome as a function of management strategy. Chest 2001; 119: 1766-77. 